\title{
A Novel Visualization Method for Sleep Spindles Based on Source Localization of High Density EEG
}

\author{
Soohyun Lee ${ }^{1}$, Seunghwan Kim ${ }^{1}$ and Jee Hyun $\mathrm{Choi}^{2 *}$ \\ ${ }^{1}$ Department of Physics, Pohang University of Science and Technology, Pohang 37673, \\ ${ }^{2}$ Center for Neuroscience, Korea Institute of Science and Technology, Seoul 02792, Korea
}

\begin{abstract}
Equivalent dipole source localization is a well-established approach to localizing the electrical activity in electroencephalogram (EEG). So far, source localization has been used primarily in localizing the epileptic source in human epileptic patients. Currently, source localization techniques have been applied to account for localizing epileptic source among the epileptic patients. Here, we present the first application of source localization in the field of sleep spindle in mouse brain. The spatial distribution of cortical potential was obtained by high density EEG and then the anterior and posterior sleep spindles were classified based on the K-mean clustering algorithm. To solve the forward problem, a realistic geometry brain model was produced based on boundary element method (BEM) using mouse MRI. Then, we applied four different source estimation algorithms (minimum norm, eLORETA, sLORETA, and LORETA) to estimate the spatial location of equivalent dipole source of sleep spindles. The estimated sources of anterior and posterior spindles were plotted in a cine-mode that revealed different topographic patterns of spindle propagation. The characterization of sleep spindles may be better be distinguished by our novel visualization method.
\end{abstract}

Key words: high density EEG, mice, sleep spindles

\section{INTRODUCTION}

A sleep spindle is a distinctive feature of electroencephalogram (EEG), which is characterized by progressively increased and gradually decreased 10 16 Hz oscillations [1]. It occurs during non-rapid eye movement (NREM) sleep and is temporally locked to the K-complex and vertex sharp wave [1]. In human EEG, sleep spindle is divided into two types, slow spindle $(\sim 12 \mathrm{~Hz})$ in the frontal and fast spindle $(\sim 14 \mathrm{~Hz})$ in parietal and central brain region [2-6]. Each type of spindle is affected differently by the

Received October 5, 2017, Revised November 30, 2017,

Accepted December 7,2017

* To whom correspondence should be addressed.

TEL: 82-2-958-6952, FAX: 82-2-958-6737

e-mail: jeechoi@kist.re.kr same variables, such as age, lifestyle and medical history [7-9]. The differences between the two types of spindles suggest that each spindle is generated by a different mechanism. However, a different mechanism of spindle generation remains unclear due to lack of spatial information of sleep spindle propagation.

Previously we reported that regionally specific sleep spindles exist in rodents, as they do in human [10]. Also, we reported corticothalamic connection among cortex, thalamic nucleus, and reticular nucleus has distinctive patterns for anterior and posterior spindles. This suggests that each type of spindle generation in the mouse had a different generation mechanism. We believe that the knowledge of rodent spindle can be an effective channel to understand human spindle. However, for mice, the comprehensive method for neuroimaging and EEG topographic measure is limited due to the small size of mouse brain. Recently, we established the source localization technique [11] in mice with the developed
Copyright ( ) Experimental Neurobiology 2017. www.enjournal.org
This is an Open Access article distributed under the terms of the Creative Commons Attribution Non-Commercial License (http://creativecommons.org/licenses/by-nc/4.0) which permits unrestricted non-commercial use, distribution, and reproduction in any medium, provided the original work is properly cited. 
high density EEG [12, 13].

In this study, we have chosen to apply our novel neuroimage technique to each type of sleep spindle and visualized the dynamics of spindles.

\section{MATERIALS AND METHODS}

We used the high density EEG data during spontaneous sleep which were collected, detected, and classified in our previous study [10]. Briefly, the high density EEG was obtained from polyimidebased microarray in freely moving mice $[12,13]$. The EEG was band-pass filtered and then the spindle detection algorithm was applied to the filtered signals based on a threshold method. The anterior and posterior spindles were classified based on K-mean clustering algorithm.

\section{Forward problem: a volume conduction model for mouse EEG}

The electrical activities inside the brain consist of currents generated by neural activities, and the major sources can be approximated to a dipole. The locations of dipoles are estimated by solving forward and inverse problems. The forward problem is to predict the distribution of electric potentials for given source locations, orientation, and signals according to Maxwell's equations, which is basically a construction of lead field matrix defining the projection from current sources at discrete locations. To reduce the errors produced by the difference between mouse head and the spherical volume conductor model, we produced a volume conduction model from in vitro magnetic resonance image (MRI) which is downloadable from an open database of the Magnetic Resonance Microimaging Neurological Atlas Group and used image data of
C57_14_M_Normal_age12 [14, 15]. (http://brainatlas.mbi.ufl. edu/Database/).

The segmentation and electrode co-registration processes were performed using Curry software (version 7, Neuroscan Inc., Charlotte, NC, USA). As the spatial resolution of Curry software is in the order of $\mathrm{mm}$, we scaled up the mouse brain by ten-fold and scaled down the conductivity parameter by ten-fold, which is mathematically right in Maxwell's equation. To co-register the electrodes' location to the MRI, we matched the positions of anatomical landmarks (nasion, preauricular left point, and preauricular right point) and the anterior and posterior commissures (AC and $\mathrm{PC}$ ). We aligned the AC-PC to a midline and estimated the coordinates of bregma. Then electrode location was co-registered by shifting from the original coordinate to the estimated bregma location.

Curry software was used to compute the boundary element method (BEM) model. To construct conduction model, we extracted cortex and white matter region from whole MRI based on brightness. We set the brain threshold as 170 and cortical threshold as 230 in 0 (black) to 255 (white) color scale. Mouse skull data is not included in MRI. Therefore, we made virtual skull layer around the brain and constructed a triangulated surfaced volume conduction model. Our BEM model consists of threelayer structures of cortex, skull, and skin. Meshes of each layer consist of triangle meshes. Cortex/skull/skin layer consisted of 1218/1301/1757 nodes and 2432/2598/3510 triangle meshes. All of the distance between nodes was maintained less than $0.5 \mathrm{~mm}$. We used conductance value as $0.033 \mathrm{~S} / \mathrm{m}$ for cortex and skin and $0.00042 \mathrm{~S} / \mathrm{m}$ for the skull.
(A)

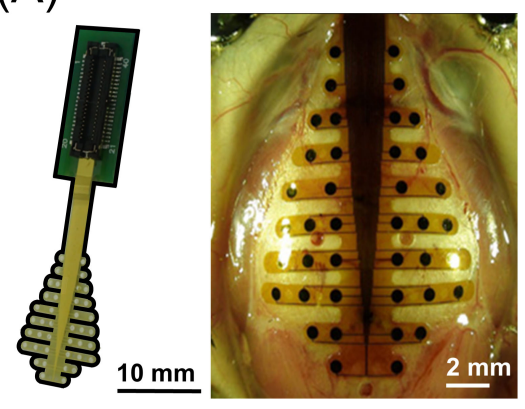

(B)

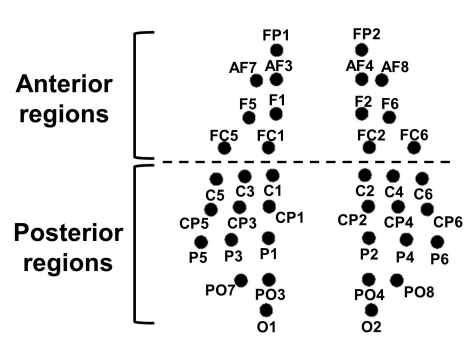

(C)

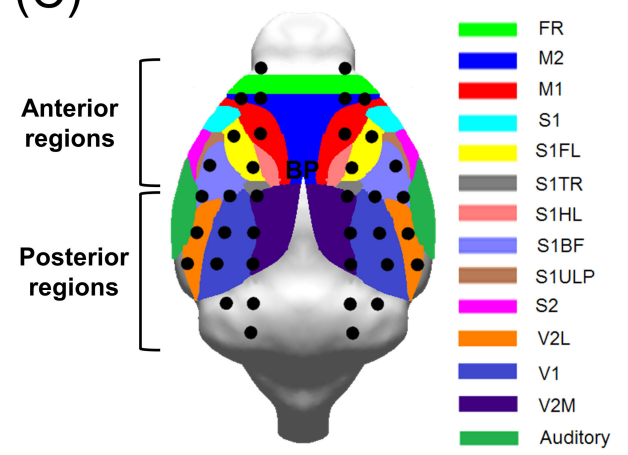

Fig. 1. Structure and montage of high density EEG. (A) Structure and size of high density EEG electrode. (B) Montage of high density EEG electrode. Based on bregma position, we divided the anterior and posterior region. The letters indicate the respective location of electrodes. (prefrontal (FP), anterior frontal (AF), frontal (F), frontocentral (FC), central (C), centroparietal (CP), parietal (P), parieto-occipital (PO) and occipital (O) position). (C) Visualization of electrode locations together with the corresponding cortical region. 


\section{Inverse problem of mouse source localization}

The goal of inverse problem is to construct the current source model, which has realistic locations and strength, from the EEG data. To solve the inverse problem and source reconstruction, we applied four source localization algorithms: minimum norm (MN) [18], low-resolution electromagnetic tomography (LORETA) [19, 20], standardized LORETA (sLORETA) [21] and exact LORETA (eLORETA) [22].

Date term $(D(j))$ and model term $(M(j))$ were given by $D(j)=\| \mathrm{L}-$ $M(j) \|^{2}$. All algorithms used same lead field matrix $(L)$, but model term was defined in a variety of ways according to algorithms. MN method used diagonal location weighting matrix. sLORETA and eLORETA is the modification of MN results. sLORETA computed statistical measure, called current strength, and eLORETA computes neuronal current flow. LORETA method included Laplacian term in model term that makes neighbored sources tend to have similar strengths.

Inverse problem has no unique solution due to lack of information. Therefore, to optimize and confirm our approach, we compared source reconstruction results to background knowledge in the case of posterior sleep spindle example.

\section{RESULTS}

We used high density EEG signals of the anterior and posterior spindles, which were clustered based on K-means cluster analysis in our previous study [10]. The representative traces of anterior and posterior spindles were presented in Fig. 2. In each panel, the left column contains the raw signals and the right column contains the filtered signals. The topographies of total power of the corresponding anterior and posterior spindles overlay the cortical surface of mouse brain in (C) and (D), respectively.

We followed the procedures for the dipole source localizations described in our previous work (Fig. 1 in [11]). Briefly, the boundary element model for the mouse brain was built based on mouse MRI to compute the leadfield matrix. Four different source localization algorithms (minimum norm [16], LORETA [17, 18], sLORETA [19] and eLORETA [20]) were applied and their performance was compared. As a demonstration, the posterior spindle shown in Fig. 2B was applied and the axial and coronal view of its estimated source distributions are shown in Fig. $3 \mathrm{~A}$ and $3 \mathrm{~B}$, respectively. While the spatial distributions of horizontal axis were similar for all algorithms, the spatial distributions of vertical axis were different depending on the algorithms. The LORETA or modified LORETA algorithms estimated the source deeper compared to MN. In our previous study based on optogenetic stimulation, the LORETA algorithm estimated the source deeper than the real source position [11]. On the other hand, the minimum norm algorithm estimated the sources successfully confined to the cortex.

The advantage of source localization is in the ability to visualize the propagation of potential sources generating the cortical
(A)

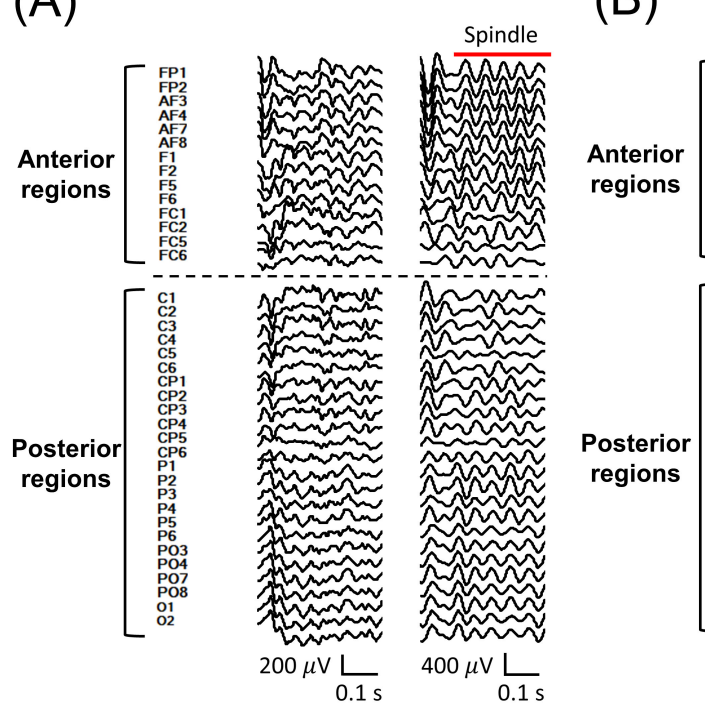

(B)

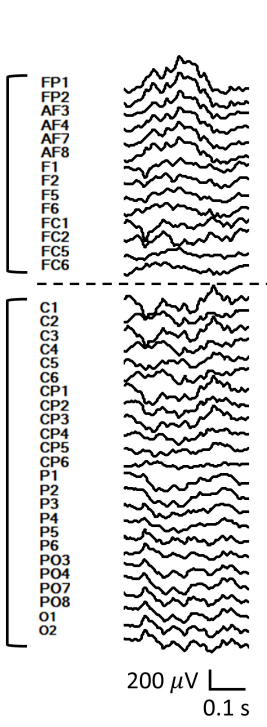

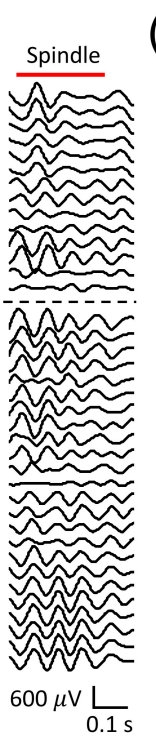

(C)

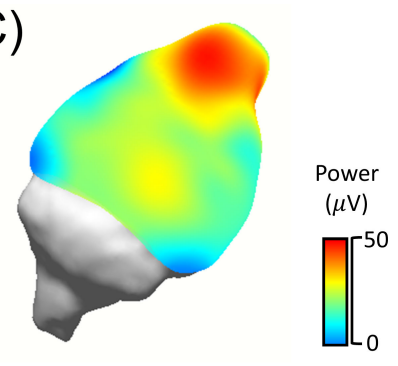

(D)

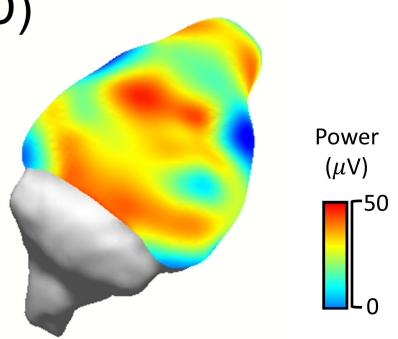

Fig. 2. High density EEG of anterior and posterior spindles. Examples of raw traces of HD-EEG (left) and filtered data (right, $8 \sim 18 \mathrm{~Hz}$ zero-phase bandpass filter) during (A) anterior and (B) posterior spindle activities. The letters indicate the electrode position as defined in Fig. 1. The topographies of averaged powers of band-pass filtered data are presented on the cortical surface of MRI for the (C) anterior and (D) posterior spindle activities. The red bar indicates spindle period. 
(A)

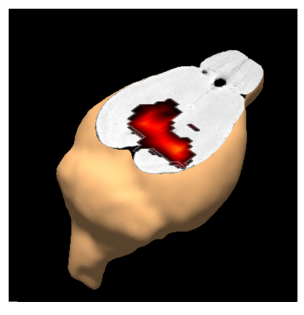

Minimum norm

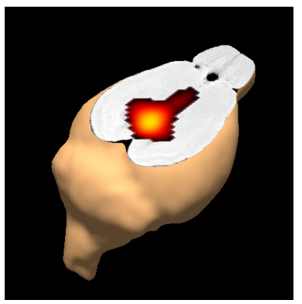

sLORETA

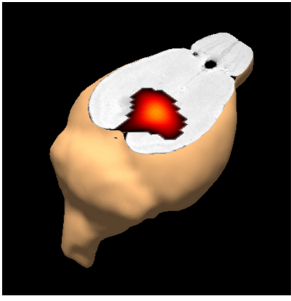

eLORETA

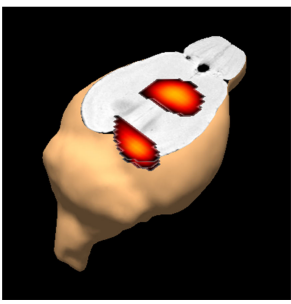

LORETA

(B)

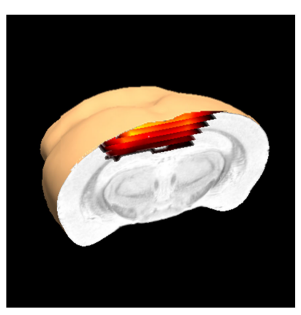

Minimum norm

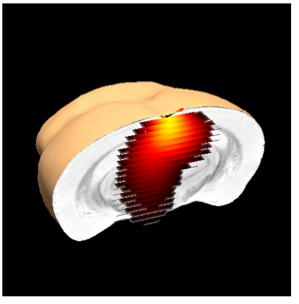

sLORETA

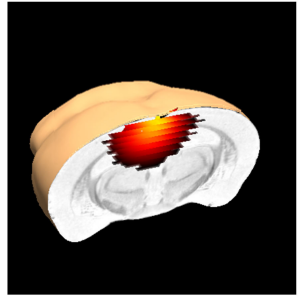

eLORETA

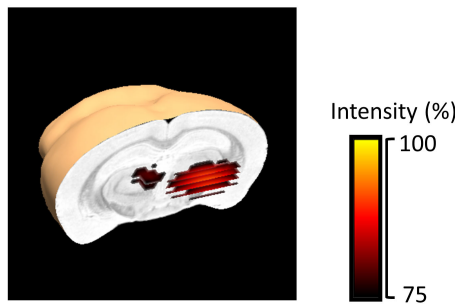

LORETA

Fig. 3. Comparing between source localization algorithms. (A) Axial view and (B) coronal view of source estimation of the posterior spindle. Axial view showed very similar patterns, but coronal view showed quite different. Except for minimum norm (MN), other method estimated the deep subcortical source. Brightness indicates the ratio to the maximum intensity of each algorithm.
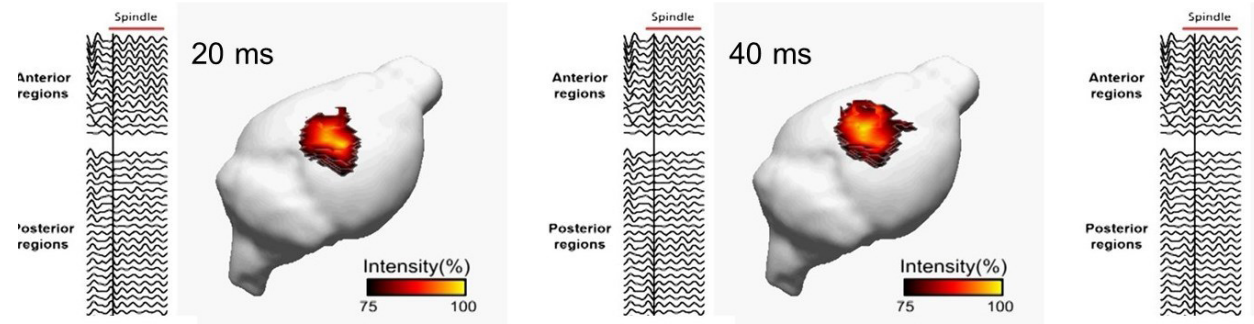

$60 \mathrm{~ms}$
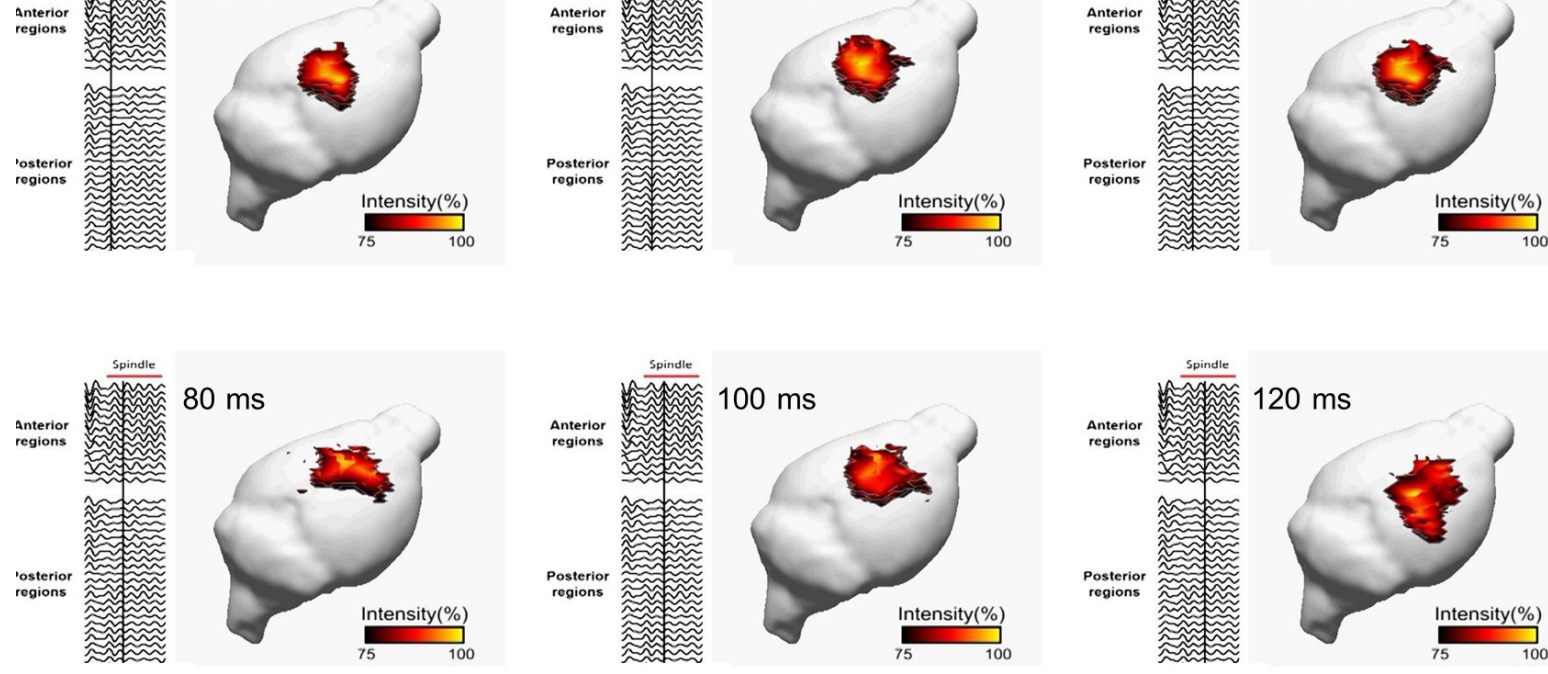

$120 \mathrm{~ms}$
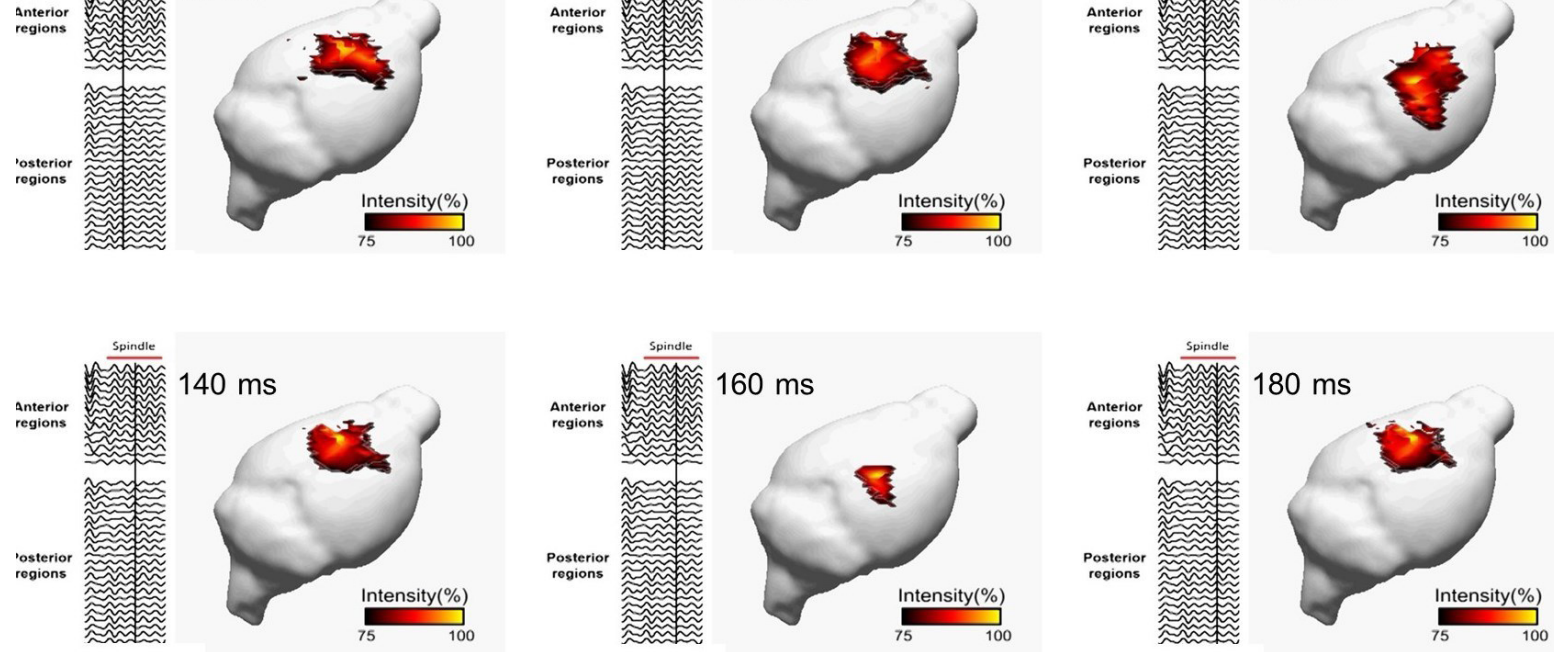

Fig. 4. Cine-mode presentation of the anterior spindles at $20 \mathrm{~ms}$ time window. Brightness indicates the ratio to the maximum intensity. 

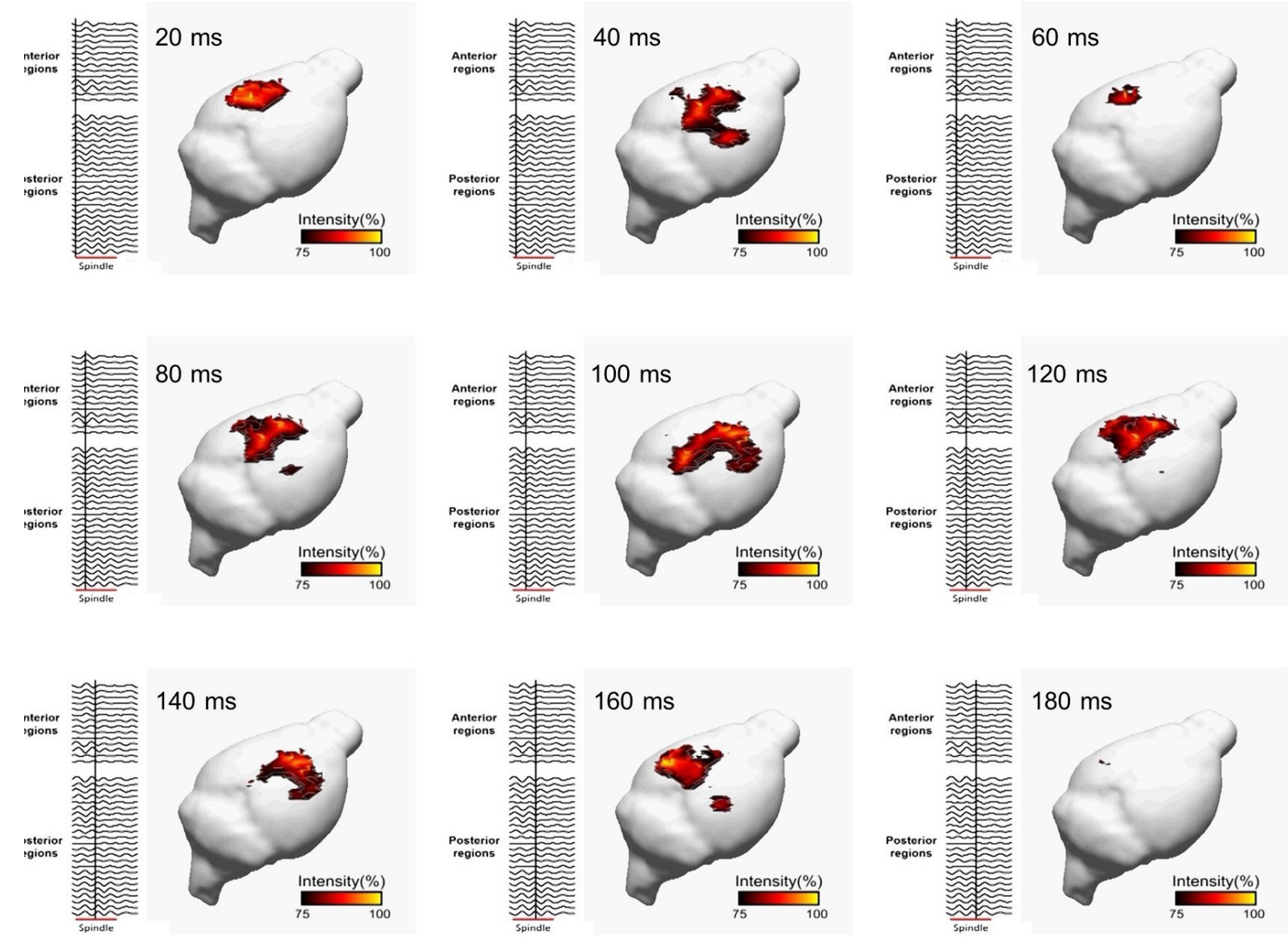

Fig. 5. Cine-mode presentation of the posterior spindles at $20 \mathrm{~ms}$ time window. Brightness indicates the ratio to the maximum intensity.

potentials. Therefore, we employed the weighted norm algorithm to sleep spindles to visualize the propagation of cortical sources as time passes and presented in cine mode for anterior and posterior sleep spindles in Fig. 4 and Fig. 5, respectively. The cine mode presentation shows the spatio-temporal patterns of spindle evolution over time. For example, the sources of posterior spindles were confined to the posterior regions, but their positions fluctuated within the posterior cortex. A movie with high temporal resolution showed that the first slow wave of spindles was generated in the somatosensory cortex and propagated to the motor cortex, but the last slow wave of spindle was generated in the motor cortex and then propagated to the somatosensory cortex, showing complex patterns of onset and propagation of sleep spindles. This visualization confirms that the spindles are dynamical phenomena rather than an event of one entity.

\section{DISCUSSION}

In this study, we evaluated the performance of four different algorithms for source localization of spindles. The flow direction of source cluster was similar, but LORETA or modified LORETA algorithms overshoot the depth of source. Generally, cortical dipole was regarded as the source of EEG [21]. Many studies have suggested that the rodent EEG has identical characteristics as the human EEG $[10,22]$. Also, an electrode with the impedance of several hundred ohms can only measure the signal in 250 $\mu \mathrm{m}$ [23]. Additionally, we used common average reference. This method cancels out the signal from deep brain region. Therefore, the subcortical source was not reasonable. One of the major possible sources of error is the electrode position. All our electrodes were located above the upper surface. Therefore, depth of source localization solution could be biased. The $\mathrm{MN}$ is used to analyze evoked responses that involve wide-spread neuronal activation over time. In addition, $\mathrm{MN}$ tends to reconstruct sources that are superficial $[24,25]$. Therefore, we recommended that MN method is suitable for mouse brain.

As a comparable tool for noninvasive functional brain mapping, functional magnetic resonance imaging (fMRI) has been used in human brain mapping. However, the fMRI is based on blood oxygen level dependenet level rather than neural activity like EEG. 
EEG is the sum of synchronized post-synaptic activities, whereas BOLD response represents the change of hemodynamics that was related to the total synaptic activity. Additionally, EEG and the BOLD signal were caused by different cellular populations [26]. Previous research shows that average distance between local field potential and centroid of functional MRI was $1 \mathrm{~cm}$ in half of the monkey sensory cortex recording [27]. As previously mentioned, EEG is more related to functional neural activity and have a better temporal resolution to understand fast brain dynamics. Besides, the temporal resolution of fMRI is inappropriate to trace the fast activities like sleep spindles.

In sum, this study employed the equivalent dipole source estimation method in visualizing the sleep spindles in mouse brain. By applying source localization method, we have shown that the anterior and posterior spindles do not have identical functional brain mapping and temporal change of spindle dynamics. Our result suggests the possibility of the minimally invasive functional approach of the spindle network analysis. Also, our approach may advance our understanding for the functional study of cortical network in mice.

\section{ACKNOWLEDGEMENTS}

This research was supported by the National Research Council of Science and Technology of Korea on project Development of Solution for Diagnosis, Treatment and Care system of Dementia (CRC-15-04-KIST), the National Research Foundation of Korea grant funded by the Korea government (2017R1A2B3012659).

\section{REFERENCES}

1. De Gennaro L, Ferrara M (2003) Sleep spindles: an overview. Sleep Med Rev 7:423-440.

2. Anderer P, Klösch G, Gruber G, Trenker E, Pascual-Marqui RD, Zeitlhofer J, Barbanoj MJ, Rappelsberger P, Saletu B (2001) Low-resolution brain electromagnetic tomography revealed simultaneously active frontal and parietal sleep spindle sources in the human cortex. Neuroscience 103:581-592.

3. Jobert M, Poiseau E, Jähnig P, Schulz H, Kubicki S (1992) Topographical analysis of sleep spindle activity. Neuropsychobiology 26:210-217.

4. Schabus M, Dang-Vu TT, Albouy G, Balteau E, Boly M, Carrier J, Darsaud A, Degueldre C, Desseilles M, Gais S, Phillips C, Rauchs G, Schnakers C, Sterpenich V, Vandewalle G, Luxen A, Maquet P (2007) Hemodynamic cerebral correlates of sleep spindles during human non-rapid eye movement sleep. Proc Natl Acad Sci U S A 104:13164-13169.
5. Werth E, Achermann P, Dijk DJ, Borbély AA (1997) Spindle frequency activity in the sleep EEG: individual differences and topographic distribution. Electroencephalogr Clin Neurophysiol 103:535-542.

6. Zygierewicz J, Blinowska KJ, Durka PJ, Szelenberger W, Niemcewicz S, Androsiuk W (1999) High resolution study of sleep spindles. Clin Neurophysiol 110:2136-2147.

7. Ayoub A, Aumann D, Hörschelmann A, Kouchekmanesch A, Paul P, Born J, Marshall L (2013) Differential effects on fast and slow spindle activity, and the sleep slow oscillation in humans with carbamazepine and flunarizine to antagonize voltage-dependent $\mathrm{Na}+$ and $\mathrm{Ca} 2+$ channel activity. Sleep 36:905-911.

8. Schönwald SV, Carvalho DZ, de Santa-Helena EL, Lemke N, Gerhardt GJ (2012) Topography-specific spindle frequency changes in obstructive sleep apnea. BMC Neurosci 13:89.

9. Tamaki M, Matsuoka T, Nittono H, Hori T (2008) Fast sleep spindle (13-15 hz) activity correlates with sleep-dependent improvement in visuomotor performance. Sleep 31:204-211.

10. Kim D, Hwang E, Lee M, Sung H, Choi JH (2015) Characterization of topographically specific sleep spindles in mice. Sleep 38:85-96.

11. Lee C, Oostenveld R, Lee SH, Kim LH, Sung H, Choi JH (2013) Dipole source localization of mouse electroencephalogram using the fieldtrip toolbox. PLoS One 8:e79442.

12. Choi JH, Koch KP, Poppendieck W, Lee M, Shin HS (2010) High resolution electroencephalography in freely moving mice. J Neurophysiol 104:1825-1834.

13. Lee M, Kim D, Shin HS, Sung HG, Choi JH (2011) Highdensity EEG recordings of the freely moving mice using polyimide-based microelectrode. J Vis Exp e2562.

14. Ma Y, Hof PR, Grant SC, Blackband SJ, Bennett R, Slatest L, McGuigan MD, Benveniste H (2005) A three-dimensional digital atlas database of the adult C57BL/6J mouse brain by magnetic resonance microscopy. Neuroscience 135:12031215.

15. Ma Y, Smith D, Hof PR, Foerster B, Hamilton S, Blackband SJ, Yu M, Benveniste H (2008) In vivo 3D digital atlas database of the adult $\mathrm{C} 57 \mathrm{BL} / 6 \mathrm{~J}$ mouse brain by magnetic resonance microscopy. Front Neuroanat 2:1.

16. Fuchs M, Wagner M, Köhler T, Wischmann HA (1999) Linear and nonlinear current density reconstructions. J Clin Neurophysiol 16:267-295.

17. Pascual-Marqui RD (1995) LORETA in 3D solution space. ISBET Newsl 6:22-28.

18. Wagner M, Fuchs M, Wischmann HA, Drenckhahn R, Köhler $\mathrm{T}$ (1996) Smooth reconstruction of cortical sources from 
EEG or MEG recordings. Neuroimage 3:S168.

19. Pascual-Marqui RD (2002) Standardized low-resolution brain electromagnetic tomography (sLORETA): technical details. Methods Find Exp Clin Pharmacol 24 Suppl D:5-12.

20. Pascual-Marqui RD, Lehmann D, Koukkou M, Kochi K, Anderer P, Saletu B, Tanaka H, Hirata K, John ER, Prichep L, Biscay-Lirio R, Kinoshita T (2011) Assessing interactions in the brain with exact low-resolution electromagnetic tomography. Philos Trans A Math Phys Eng Sci 369:3768-3784.

21. Niedermeyer E, da Silva FL (2005) Electroencephalography: basic principles, clinical applications, and related fields. 5th ed. Lippincott Williams \& Wilkins, Philadelphia, PA.

22. Kim B, Kocsis B, Hwang E, Kim Y, Strecker RE, McCarley RW, Choi JH (2017) Differential modulation of global and local neural oscillations in REM sleep by homeostatic sleep regulation. Proc Natl Acad Sci U S A 114:E1727-E1736.
23. Kajikawa Y, Schroeder CE (2011) How local is the local field potential? Neuron 72:847-858.

24. Dale AM, Liu AK, Fischl BR, Buckner RL, Belliveau JW, Lewine JD, Halgren E (2000) Dynamic statistical parametric mapping: combining $\mathrm{fMRI}$ and MEG for high-resolution imaging of cortical activity. Neuron 26:55-67.

25. Hämäläinen MS, Ilmoniemi RJ (1994) Interpreting magnetic fields of the brain: minimum norm estimates. Med Biol Eng Comput 32:35-42.

26. Nunez PL, Silberstein RB (2000) On the relationship of synaptic activity to macroscopic measurements: does co-registration of EEG with fMRI make sense? Brain Topogr 13:79-96.

27. Disbrow EA, Slutsky DA, Roberts TP, Krubitzer LA (2000) Functional MRI at 1.5 tesla: a comparison of the blood oxygenation level-dependent signal and electrophysiology. Proc Natl Acad Sci U S A 97:9718-9723. 